\title{
Therapeutic Use of Convalescent Plasma in COVID-19 Infected Patients with Concomitant Hematological Disorders
}

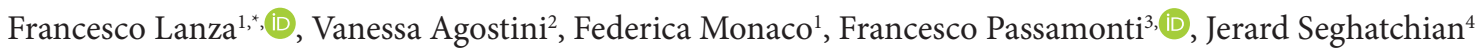 \\ ${ }^{1}$ Hematology Unit \& Romagna Transplant Network, Ravenna, Italy \\ ${ }^{2}$ Transfusion Medicine Department, IRCCS- Ospedale Policlinico San Martino, Genova, Italy \\ ${ }^{3}$ Hematology Unit, University of Insubria, Varese, Italy \\ ${ }^{4}$ International Consultancy in Innovative Manufacturing and Quality/Safety of Blood-Derived Bioproducts, London, England, UK
}

\section{ARTICLE INFO}

Article History

Received 11 February 2021

Accepted 17 March 2021

Keywords

SARS-CoV-2

COVID-19 antibodies

plasmapheresis

convalescent plasma preparations

hematological disorders

neutralising antibodies

prolonged viral shedding

COVID-19 mutation

\begin{abstract}
The use of convalescent plasma (CP) from individuals recovered from severe acute respiratory syndrome coronavirus 2 (SARS-CoV-2) is a promising therapeutic modality for the coronavirus disease 2019 (COVID-19). CP has been in use for at least a century to provide passive immunity against a number of diseases, and was recently proposed by the World Health Organization for human Ebola virus infection. Only a few small studies have so far been published on patients with COVID-19 and concomitant hematological malignancies (HM). The Italian Hematology Alliance on HM and COVID-19 has found that HM patients with COVID-19 clinically perform more poorly than those with either HM or COVID-19 alone. A COVID-19 infection in patients with B-cell lymphoma is associated with impaired generation of neutralizing antibody titers and lowered clearance of SARS-CoV-2. Treatment with CP was seen to increase antibody titers in all patients and to improve clinical response in $80 \%$ of patients examined. However, a recent study has reported impaired production of SARS-CoV-2-neutralizing antibodies in an immunosuppressed individual treated with $\mathrm{CP}$, possibly supporting the notion of virus escape, particularly in immunocompromised individuals where prolonged viral replication occurs. This may limit the efficacy of CP treatment in at least some HM patients. More recently, it has been shown that CP may provide a neutralising effect against B.1.1.7 and other SARS-CoV-2 variants, thus expanding its application in clinical practice. More extensive studies are needed to further assess the use of CP in COVID-19-infected HM patients.
\end{abstract}

(c) 2021 International Academy for Clinical Hematology. Publishing services by Atlantis Press International B.V. This is an open access article distributed under the CC BY-NC 4.0 license (http://creativecommons.org/licenses/by-nc/4.0/).

\section{INTRODUCTION}

In December 2019, an outbreak of a novel coronavirus now called severe acute respiratory syndrome coronavirus 2 (SARS-CoV-2) was reported in Wuhan, China. By mid-March 2020, the World Health Organization (WHO) had declared COVID-19, the disease caused by SARS-CoV-2, to be a global pandemic $[1,2]$.

Passive immunotherapy with plasma collected from patients recovered from SARS-CoV-2, known as convalescent plasma (CP), is a promising option for the treatment of COVID-19-infected patients. The transfusion of convalescent blood products with the goal of providing passive immunity has been in use for at least a century to treat a number of diseases ranging from polio, chickenpox, and measles, to SARS and Middle East respiratory syndrome (MERS). More recently, the WHO proposed the use of CP as an early treatment for patients affected with Ebola virus disease $[3,4]$.

Passive immunization can be achieved by using transfusions of CP in patients infected with several pathogens $[1,2,5]$. CP is widely employed in transfusion practice in countries where modern mobile apheresis technologies are available. These mobile and fully-

“Corresponding author. Email: francesco.lanza@auslromagna.it

Peer review under responsibility of the International Academy for Clinical Hematology automated devices based on digital technology can be used in both hospital and in-home care settings. Ideally, plasma is obtained from donors by plasmapheresis; alternatively, whole blood can also be collected, with plasma separation carried out at another stage of the transfusion process. The use of CP or CP components containing immunoglobulin from patients who have fully recovered from COVID-19 may therefore provide a feasible form of therapy in patients who are otherwise unable to defend themselves from the virus $[1,2]$. However, this procedure does not differentiate autoantibodies, which are not desirable, from the COVID neutralizing antibodies [6].

To meet the demand for immune plasma, Blood Banks need to establish appropriate guidelines and requirements for the donation, collection, processing, storage, testing, and distribution of the product $[1,2,7,8]$. Most authors agree with the recommended administration of $200-600 \mathrm{~mL}$ of immune plasma $(8-10 \mathrm{~mL} / \mathrm{kg})$ once a day for up to 3-7 consecutive days. However, several technical questions still need to be resolved, such as the optimal threshold of specific neutralizing antibody serum titer $(>160$ or $>320$ with the enzyme immunoassay [EIA] method) in the preparation, and how important it is to perform pathogen (viral) inactivation treatment of the plasma, in view of the presence of viral DNA in the donor population. As far as the plasma volume is concerned, Duan et al. used $200 \mathrm{~mL}$ of CP containing high neutralizing antibody titers $(>1: 640)$ for the treatment of 10 critical patients with COVID-19 (age range 
34-78) after an average of 16.5 days of admission to the hospital. $\mathrm{CP}$ treatment increased the neutralizing antibody titer, oxygen saturation and lymphocyte count in the examined individuals; CP treatment was further associated with a reduction in SARS-CoV-2 viral load and lung lesions. While there was no significant difference in clinical characteristics between patients in the CP-treated and the control groups, the results in the former were superior $(p<0.001)$ [9]. Looking at the data so far published in the literature, there is no consensus regarding the optimal plasma volume and antibody titers, to be used for a successful CP preparation.

The use of CP and/or plasmapheresis in the low- and mediumincome part of the world is another interesting issue. Although plasmapheresis is feasible in medium income nations, advanced methodologies for CP preparations are less likely to be proposed and implemented in poor countries [10,11].

In most circumstances, the SARS-CoV-2 antibodies detected by enzyme linked immunosorbent assay (ELISA) assays in plasma samples bind to several different receptors, including SARS-CoV-2 spike ectodomain (S/ECD) and receptor-binding domain (S/RBD) proteins. Those are able to neutralize SARS-CoV-2 in vitro and in vivo, thus providing valuable information about their potential use as surrogates for virus neutralization $[8,9]$. The possible mechanisms of action and the consequences of $\mathrm{CP}$ treatment on the pathogenesis of COVID-19 include direct neutralization of the virus, control of overactive immune functions, such as cytokine storm, Th1/Th17 ratio, complement activation, hyperinflammation, immunomodulation and a hypercoagulable state, and, possibly, reduce in the incidence of pulmonary embolism. The occurrence of infection-induced complement activation in the early stage of the disease and the formation of some autoantibodies in some cases represent further elements to be considered in this setting $[8,9,12]$.

The overall benefits of COVID-19 CP are expected to be better achieved in non-critical hospitalized and in homecare patients $[6,12]$. One of the most important mechanisms underlying the deterioration of disease leading to organ injury is cytokine storm. Therapies such as interleukin- 6 antibody blocker, stem cell therapy, and transfusion of $\mathrm{CP}$ are currently being used with considerable success in countering this uncontrollable inflammatory response $[1,13]$.

So far, five randomized studies have been published on the role of CP in COVID-19. Li et al. investigated 103 patients and found that using CP therapy alongside standard treatment in patients with severe or life-threatening COVID-19 provided no clinical benefit over standard treatment alone in the 28-day follow-up period of the study. However, the analysis did not consider the potential benefits of CP administration over the longer-term (3-6 months) [14].

In a more recent study involving 160 randomized patients, early administration (within $72 \mathrm{~h}$ after the onset of mild COVID-19 symptoms) of CP containing high titers of anti-SARS-CoV-2 antibodies was associated to better outcomes than in mildly ill infected older adults without treatment [1].

Interestingly, based on a careful evaluation of both randomized and observational studies, the Food and Drug Administration granted an emergency use authorization for CP in hospitalized COVID-19 infected patients with signs of progressive disease. Similar indication was given by the American Association of Blood Banks, which suggested an early use (preferably within 3 days after coronavirus diagnosis) of CP.
A recent meta-analysis of randomized clinical trials and matched-control data showed that the use of CP was associated with a lower mortality rate compared to patients treated with standard drugs. Moreover, a lower mortality was demonstrated in patients who were treated with high-titer CP within 3 days of hospital admission [16].

This evidence suggests that COVID-19 patients with mild symptoms and those not yet undergoing noninvasive ventilation may be excellent candidates for passive immunotherapy. Further randomized clinical trials addressing the clinical usefulness of CP therapy in various patient subcategories and at different stages of the disease are needed to better elucidate this field of investigation $[1,2]$. In particular, the optimal window for donating high neutralizing titer CP for COVID-19 immunotherapy is unknown. Careful studies must therefore be made of the response kinetics of antibodies against the SARS-CoV-2 surface spike glycoprotein and in vitro live virus neutralizing $(\mathrm{VN})$ titers (Table 1 ).

\section{METHODOLOGICAL ADVANCEMENT}

There is an accumulating body of evidence that the development of novel and innovative strategies related to the use of CP may expand its clinical applications in transfusion medicine. For example, several methodological improvements in the use of the ultraviolet $\mathrm{C}$ (UVC), sterilization of fresh frozen plasma (FFP)/cryosupernatantdepleted FFP or immunoglobulins containing neutralizing antibodies for clinical use against COVID-19 may be of clinical relevance, possibly impacting on the outcome of patients treated with newer CP preparations. It bears pointing out that some transfusion services with the appropriate skills in handling therapeutic apheresis may already have the appropriate know-how to achieve this goal. A protocol based on the use of cryosupernatant may represent a safer approach for this purpose, since the large majority of the immune complexes and IgM bound to virus and high molecular-weight cryoglobulin are removed, while other essential components of plasma that might have some antiviral and antiinflammatory properties would remain in the cryo-supernatant.

Another validated method that could improve the quality of FFP plasmapheresis and its derived supernatant involves pathogen reduction technology, based on a sterilization process to eliminate viruses and bacteria contaminating the blood product, as well as the use of purer affinity column derived specific antibodies [8]. Moreover, the CP can be collected using a selective apheresis process to avoid the unnecessary loss of red cells in the donor and to optimize the volume of plasma that can be produced. This can be performed by expert technicians using double or triple plasmapheresis technologies in a safe environment. All of these methods need to meet the standard selection criteria for plasma donation in compliance with national legislation and standard operational procedures. The requirement for limiting the risk of Transfusion Related Acute Lung Injury (TRALI) can be met by using plasma from male donors or from female donors with no history of pregnancy. Such measures are known to significantly decrease the presence in the plasma of antibodies to HLA or granulocyte antigens that can lead to TRALI.

Another phenomenon which may arise following the administration of CP is antibody-dependent enhancement. In this case, antibodies against SARS-CoV-2 may bind to the virus without neutralizing it, thus enhancing viral entry into cells and viral 
Table 1 List of available therapies for COVID-19

\begin{tabular}{lll}
\hline Drug & $\begin{array}{l}\text { EMA approved in clinical practice } \\
\text { for hospitalizated patient }\end{array}$ & Clinical trial \\
\hline Hydroxychloroquine & No & Yes, local study \\
Azithromycin & No & No \\
Darunavir/cobicistat & No & Yes, radomized study \\
Lopinavir/ritonavir & No & Yes, radomized study \\
Remdesivir & Yes (in selected cases) & \\
Low molecular weight heparins & Yes (first line) & \\
Corticosteroids & Yes (first line) & Meta-analysis review \\
Ivermectin & No & Yes, clinical study \\
Siltuximab (moAB- anti IL6) & No & Yes, clinical study \\
Tocilizumab, sarilumumab (anti R-IL6) & No & Yes, clinical study \\
Anakinra (anti IL1) & No & Yes, clinical study \\
Alpha/beta INF & No & Yes, clinical study \\
Baricitinib (JAK1/2 inibitors) & No & Yes, clinical study \\
Ruxolitinib or Tofacitinib (JAK2 inibitors) & No & Yes, clinical study \\
Acalabrutinib, ibrutinib, zanobrutinib (BTK inibitors) & No & Yes, clinical study \\
Bamlanivimab & No & Yes, clinical study \\
Casirivimab plus Imdevimab & No & Yes, clinical study \\
Non-SARS-CoV-2-specific intravenous immune & Yes (in selected cases) & \\
globulin (IVIG) & & \\
Convalescent plasma & No & Yes, in randomized study \\
Autologous hematopoietic stem cells & No & Yes, local study \\
Mesenchymal stem cells & No & Yes, clinical study \\
\hline
\end{tabular}

EMA, European Medicine Agency. (See References [39-43]).

replication. This phenomenon has been described in Dengue Virus as well as in several species of coronaviruses in "in vitro" cell culture experiments $[5,6]$.

A critical issue in the preparation of $\mathrm{CP}$ is how much plasma needs to be collected. Based on recently published data [5,7], the reference target ranges from 200 to $600 \mathrm{~mL}$ (without anticoagulant), according to local requirements and national legislation. The interval between the first plasma donation and the subsequent ones is dictated by local regulations, but is strictly dependent on the donors' health status.

Pathogen inactivation is a controversial issue related to the treatment of plasma once collected from the donor. In some countries, such as Italy, this procedure is mandatory, but in others it is optional. We believe that pathogen inactivation is the best way of ensuring the safety of using plasma components, and that it should be deemed an essential regulatory requirement to limit the risk of spreading infectious diseases through transfusion, which would pose particularly serious risks to COVID-19 infected patients.

Moreover, the use of an on-line affinity column may make it possible to remove the COVID-19 virus and its antibodies from the blood of both donor and recipient. Greater understanding of the persistence of RBD and ECD IgG and IgM levels over a period of 3-6 months post infection and of the kinetics of SARS-CoV-2 neutralizing titers may lead to improvements in the preparation of CP units.

In our view, a better and more modern take on CP protocols is based on affinity column-derived neutralizing antibody hyperconcentrate, which can be obtained reliably and consistently from CP. This method has the advantage of increasing the quantity of neutralizing antibodies in the circulation without delay and spontaneously reducing the rate of infectivity, thus indirectly encouraging herd immunity.

\section{USE OF CONVALESCENT PLASMA IN PATIENTS WITH HEMATOLOGICAL DISORDERS AND ITS PUTATIVE ROLE IN SARS-COV-2 VARIANTS}

Another highly important issue to be addressed is the role played by plasmapheresis in immunocompromised patients, particularly in those with hematological malignancies (HM). Since the morbidity and mortality associated with coronavirus are highest in the elderly and among individuals with underlying co-morbidities, it would be of great interest to identify the disease categories which are most susceptible to COVID-19 infection, in order to recommend specific therapeutic interventions. Several recentlypublished [17-19], small-scale studies have shown that patients with COVID-19 and concomitant HM have a higher mortality rate than uninfected patients with HM. A recent report by the Italian Hematology Alliance on COVID-19 demonstrated that patients with HM have better outcomes than both the general population with COVID-19 and patients with HM and concomitant COVID-19 [20]. It would also be useful to carry out a full clinical assessment of patients treated with hematopoietic stem cell transplantation (HSCT) in the current COVID-19 era. Approximately 50,000 HSCT procedures are performed annually with good survival outcomes, so it would be useful to assess the real risk of COVID-19 infection and susceptibility to severe pulmonary distress and death in such a large population [21-23].

A report on a lymphoma patient who underwent HSCT showed that, following transplantation, COVID-19 infection was associated with persistent SARS-CoV-2 viral shedding, which lasted for 74 days and was unresponsive to the administration of CP [24]. Another study demonstrated that the use of $\mathrm{CP}$ in patients with $\mathrm{HM}$ was associated with an improved 30-day survival in comparison with untreated controls, thus confirming its utility in this clinical setting [25]. 
The in vivo use of CD20 MoAbs, such as rituximab, is associated with a marked reduction of circulating B-lymphocytes, thus negatively affecting anti-viral immunity. As a consequence, COVID-19 positive patients previously treated with CD20 MoAbs may produce fewer SARS-CoV-2 neutralizing antibodies, run a higher risk of COVID-19 reinfection once recovered, and could respond poorly to vaccines. In two recent studies, protracted cases of COVID-19 disease were observed in patients with elevated B-lymphocyte counts. These studies found a positive correlation between SARS-CoV-2 RNAaemia and prior use of anti-CD20 MoAbs [26,27]. Interestingly, Kemp et al. demonstrated both in vitro and in vivo that some spike mutations are associated with prolonged COVID-19 persistence in affected patients [28]. These mutations conferred lower sensitivity to $\mathrm{CP}$ therapy, suggesting that spike escape variants may be a factor in unresponsiveness to $\mathrm{CP}$ and prolonged SARS-CoV-2 infection.

In contrast, Montero-Escribano et al. [29] found no increased risk of SARS-CoV-2 infection in 60 patients with multiple sclerosis receiving anti-CD20 therapy. This indicates that the patient's underlying disease, as well as the cumulative dosage of CD20 MoAbs, may be critical factors in determining the viral clearance of SARSCoV-2 and potential severity of disease if contracted.

A study of patients who received kidney transplantation showed a higher proportion of severe COVID-19 pneumonia than in the general population. However, despite the severity and protracted clinical course of infection, virus shedding was associated with full recovery, arguing against the risk of a poor prognosis [30].

It is well known that COVID-19-related pneumonia is mediated by hyper-activation of effector $\mathrm{T}$ cells and excessive production of inflammatory cytokines, such as IL-6, IL-1, interferon-gamma, and tumor necrosis factor (TNF). This inflammatory process may cause a pathological process resulting in plasma leakage, vascular permeability, and disseminated intravascular coagulation. Known as "cytokine storm", this reaction is a life-threatening complication of COVID-19 infection. The immunocompromised status associated with HM may enhance the risk of bacterial sepsis and of viral infections such as COVID-19.
The preventive or therapeutic use of CP may therefore be beneficial in chemotherapy-treated cancer patients, possibly mitigating the impact of COVID-19 [31]. However, the incidence and potential predictive parameters of mortality of COVID-19 in patients with HM is still a matter of investigation [19,20]. Moreover, different gene responses, types of immunities, underlying conditions, gender-related lifestyle behaviours, and races could increase the risk for COVID-19-related complications. Furthermore, it has been demonstrated that the SARS-CoV-2 spike protein is critical for virus infection through engagement of the angiotensin converting enzyme 2 (ACE2) receptor, and therefore the occurrence of amino acid variation in the spike portion of the virus may play a role in determining disease severity. A recent report has shown fatal SARS-CoV-2 escape from neutralizing antibodies in an immunesuppressed individual treated with $\mathrm{CP}$, suggesting its limited activity in patients with HM $[31,32]$.

It is our view that the clinical use of CP may play a certain role in the treatment of critically ill patients with severe respiratory distress syndrome or septic shock associated with COVID-19 infection. Nonetheless, responsiveness may vary at different stages of the disease. Recent investigations indicate that early-intermediate stages of the cytokine storm reaction phase may be more responsive to CP [31,32]. Moreover, COVID-19 infected patients who showed a high incidence of thrombosis along with related laboratory abnormalities responded successfully to $\mathrm{CP}$, with significant improvements in coagulation status [6,33].

Recent studies have shown that blood transfusions can make individuals more vulnerable against COVID-19 infection as a result of side effects such as iron overload, alloimmunization, and transfusion associated infections $[3,5]$. These findings may have implications in immunocompromised patients with HM and concomitant COVID-19 infection. Further work is required to better assess the clinical implications related to the presence or absence of neutralizing antibodies as well as the duration of COVID-19 infection in the host and to better predict the frequency of COVID-19 re-infection and responsiveness to vaccination strategies [34] (Figure 1).

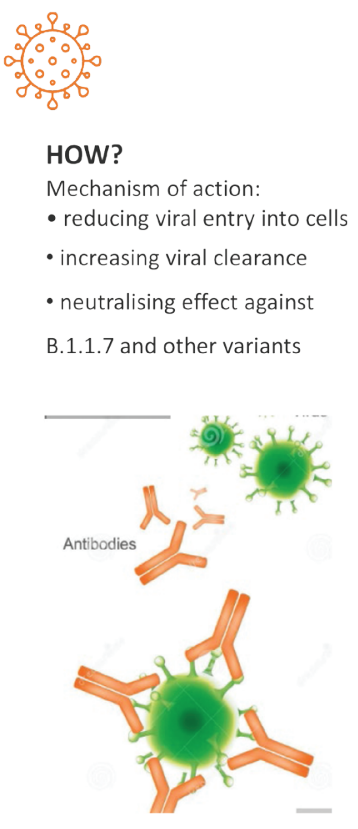

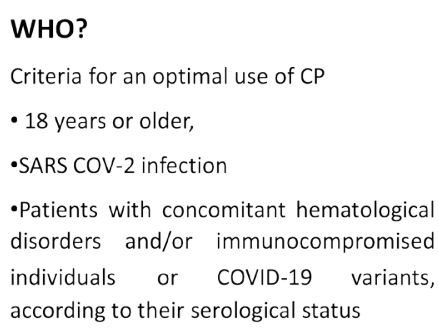

WHEN?

EARLY phases (before critical disease develops):

$\downarrow$ the duration of infection,

the development of symptoms,

the development of severe disease requiring intubation

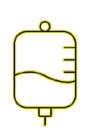

\begin{tabular}{lc}
\multicolumn{2}{l}{ SAFETY } \\
SAE & $\% *$ \\
Mortality within four hours of transfusion & 0.05 \\
TACO & 0.18 \\
TRALI & 0.10 \\
Severe allergic transfusion reaction & 0.10 \\
7-day SAE reports & \\
Thrombolic or thromboembolic complication & 0.19 \\
Sustained hypotension & 0.27 \\
Cardiac events & 0.40
\end{tabular}

NO LATE $\rightarrow$ late-stage disease is not beneficial

Figure 1 Indications for the use of convalescent plasma for COVID-19-infected subjects. (See References [15,31,38]). 


\section{CONCLUDING REMARKS}

This paper has discussed the potential usefulness of CP applications in transfusion medicine, and suggests that methodological improvements may be of clinical value to COVID-19 infected patients, potentially enhancing immunomodulation and reducing hyper-inflammatory states. The hyperconcentrate preparation of CP appears to be useful, since it can also be administered to individuals who are poor responders to the COVID-19 vaccine and can provide an immediate neutralizing antibody response to those who are in need. Further clinical studies will help clarify the role played by CP in the immunocompromised host with COVID-19 infection and concomitant HM and will elucidate the timing (post-exposure prophylaxis, early use, late use) for its optimal use [35-37].

\section{CONFLICTS OF INTEREST}

The authors declare they have no conflicts of interest.

\section{AUTHORS' CONTRIBUTION}

FL designed the review article, wrote the first draft and reviewed and approved the final manuscript. JS wrote a portion of the methodological advancement section. VA, FM and FP critically reviewed the manuscript and scrutinized results from the published material. FM edited figure and table. All authors approved the final manuscript.

\section{ACKNOWLEDGMENTS}

We thank "Associazione Italiana Leucemie" (AIL-Ravenna) and GIIMA Society for the support.

\section{REFERENCES}

[1] Shen C, Wang Z, Zhao F, Yang Y, Li J, Yuan J, et al. Treatment of 5 critically ill patients with COVID-19 with convalescent plasma. JAMA 2020;323;1582-9.

[2] Casadevall A, Pirofski LA. The convalescent sera option for containing COVID-19. J Clin Invest 2020;130;1545-8.

[3] World Health Organization. Use of convalescent whole blood or plasma collected from patients recovered from Ebola virus disease for transfusion as an empirical treatment during outbreaks. 2014, pp. 1-19. Available from: http://apps.who.int/iris/rest/bitstreams/604045/retrieve (accessed February 20, 2020).

[4] Burnouf T, Seghatchian J. Ebola virus convalescent blood products: where we are now and where we may need to go. Transfus Apher Sci 2014;51;120-5.

[5] Seghatchian J, Lanza F. Convalescent plasma, an apheresis research project targeting and motivating the fully recovered COVID-19 patients: a rousing message of clinical benefit to both donors and recipients alike. Transfus Apher Sci 2020;59;102794.

[6] Amiral J, Vissac AM, Seghatchain J. COVID-19, induced activation of haemostasis, and immune reactions: can an auto-immune reaction contribute to the delayed severe complications observed in some patients?. Transfus Apher Sci 2020;59;102804.
[7] Accorsi P, Berti P, de Angelis V, De Silvestro G, Mascaretti L, Ostuni A, et al. Position paper on the preparation of immune plasma to be used in the treatment of patients with COVID-19. Transfus Apher Sci 2020;59;102817.

[8] Lanza F, Seghatchian J. Reflection on passive immunotherapy in those who need most: some novel strategic arguments for obtaining safer therapeutic plasma or autologous antibodies from recovered COVID-19 infected patients. Br J Haematol 2020;190; e27-e9.

[9] Duan K, Liu B, Li C, Zhang H, Yu T, Qu J, et al. Effectiveness of convalescent plasma therapy in severe COVID-19 patients. Proc Natl Acad Sci U S A 2020;117;9490-6.

[10] Leemhuis T, Padley D, Keever-Taylor C, Niederwieser D, Teshima T, Lanza F, et al. Essential requirements for setting up a stem cell processing laboratory. Bone Marrow Transplant 2014;49; 1098-105.

[11] Smit Sibinga CT, Abdella YE, Seghatchian J. Poor economics transforming challenges in transfusion medicine and science into opportunities. Transfus Apher Sci 2020;59;102752.

[12] Franzè E, Stolfi C, Troncone E, Scarozza P, Monteleone G. Role of Interleukin-34 in cancer. Cancers (Basel) 2020;12;252.

[13] Monteleone G, Sarzi-Puttini PC, Ardizzone S. Preventing COVID-19-induced pneumonia with anticytokine therapy. Lancet Rheumatol 2020;2;e255-e6.

[14] Li L, Zhang W, Hu Y, Tong X, Zheng S, Yang J, et al. Effect of convalescent plasma therapy on time to clinical improvement in patients with severe and life-threatening COVID-19: a randomized clinical trial. JAMA 2020;324;460-70.

[15] Libster R, Pérez Marc G, Wappner D, Coviello S, Bianchi A, Braem V, et al. Early high-titer plasma therapy to prevent severe Covid-19 in older adults. N Engl J Med 2021;384;610-18.

[16] Klassen SA, Senefeld JW, Johnson PW, Carter RE, Wiggins CC, Shoham S, et al. The effect of convalescent plasma therapy on COVID-19 patient mortality: systematic review and metaanalysis. Mayo Clin Proc 2021 (in press).

[17] Lanza F, Saccardi R, Seghatchian J. New horizons on stem cell cryopreservation through the artificial eyes of CD 34+, using modern flow cytometry tools. Transfus Apher Sci 2020;59;102785.

[18] Hu Y, Wang L, Cui Q, Tan Su Yin E, Wu H, Nagler A, et al. COVID-19 in hematologic malignancies: big challenges. Clin Hematol Int 2020;2;173-5.

[19] Brissot E, Labopin M, Baron F, Bazarbachi A, Bug G, Ciceri F, et al. Management of patients with acute leukemia during the COVID-19 outbreak: practical guidelines from the acute leukemia working party of the European Society for Blood and Marrow Transplantation. Bone Marrow Transplant 2021;56;532-5.

[20] Passamonti F, Cattaneo C, Arcaini L, Bruna R, Cavo M, Merli F, et al. Clinical characteristics and risk factors associated with COVID-19 severity in patients with haematological malignancies in Italy: a retrospective, multicentre, cohort study. Lancet Haematol 2020;7;e737-e45.

[21] Dholaria B, Savani BN. How do we plan hematopoietic cell transplant and cellular therapy with the looming COVID-19 threat?. Br J Haematol 2020;189;239-40.

[22] Algwaiz G, Aljurf M, Koh M, Horowitz MM, Ljungman P, Weisdorf D, et al. Real-world issues and potential solutions in hematopoietic cell transplantation during the COVID-19 pandemic: perspectives from the Worldwide Network for Blood and Marrow Transplantation and Center for International Blood and Marrow Transplant Research Health Services and 
International Studies Committee. Biol Blood Marrow Transplant 2020;26;2181-9.

[23] Sharm A, Bhatt NS, St Martin A, Bilal Abid M, Bloomquist J, Chemaly RF. Clinical characteristics and outcomes of COVID-19 in haematopoietic stem-cell transplantation recipients: an observational cohort study. Lancet Haematol 2021;8;e185-e93.

[24] Karataş A, İnkaya AÇ, Demiroğlu H, Aksu S, Haziyev T, Çınar OE, et al. Prolonged viral shedding in a lymphoma patient with COVID-19 infection receiving convalescent plasma. Transfus Apher Sci 2020;59;102871.

[25] Thompson MA, Henderson JP, Shah PK, Rubinstein SM, Joyner MJ, Choueiri TK, et al. Convalescent plasma and improved survival in patients with hematologic malignancies and COVID-19. medRxiv 2021.

[26] Ferrari S, Caprioli C, Weber A, Rambaldi A, Lussana F. Convalescent hyperimmune plasma for chemo-immunotherapy induced immunodeficiency in COVID-19 patients with hematological malignancies. Leuk Lymphoma 2021;1-9.

[27] Hueso T, Pouderoux C, Péré H, Beaumont AL, Raillon LA, Ader F, et al. Convalescent plasma therapy for B-cell-depleted patients with protracted COVID-19. Blood 2020;136;2290-5.

[28] Kemp SA, Collier DA, Datir R, Ferreira IATM, Gayed S, Jahun A, et al. Neutralising antibodies in Spike mediated SARS-CoV-2 adaptation. medRxiv 2020.

[29] Montero-Escribano P, Matías-Guiu J, Gómez-Iglesias P, PortaEtessam J, Pytel V, Matias-Guiu JA. Anti-CD20 and COVID-19 in multiple sclerosis and related disorders: a case series of 60 patients from Madrid, Spain. Mult Scler Relat Disord 2020;42;102185.

[30] Zhu L, Gong N, Liu B, Lu X, Chen D, Chen S, et al. Coronavirus disease 2019 pneumonia in immunosuppressed renal transplant recipients: a summary of 10 confirmed cases in Wuhan, China. Eur Urol 2020;77;748-54.

[31] Betrains A, Godinas L, Woei-A-Jin FJSH, Rosseels W, Van Herck Y, Lorent N, et al. Convalescent plasma treatment of persistent severe acute respiratory syndrome coronavirus-2 (SARS-CoV-2) infection in patients with lymphoma with impaired humoral immunity and lack of neutralizing antibodies. Br J Haematol 2021;192;1100-5.

[32] Abu Jabal K, Ben-Amram H, Beiruti K, Batheesh Y, Sussan C, Zarka $\mathrm{S}$, et al. Impact of age, ethnicity, sex and prior infection status on immunogenicity following a single dose of the BNT162b2 mRNA COVID-19 vaccine: real-world evidence from healthcare workers, Israel, December 2020 to January 2021. Euro Surveill 2021;26;2100096.

[33] Joyner JM, Carter RE, Senefeld JW, Klassen SA, Mills JR, Johnson PW, et al. Convalescent plasma antibody levels and the risk of death from Covid-19. N Engl J Med 2021;384;1015-27.

[34] Shenoy AG, Hettinger AZ, Fernandez SJ, Blumenthal J, Baez V. Early mortality benefit with COVID-19 convalescent plasma: a matched control study. Br J Haematol 2021;192;706-13.

[35] Lanza F, Seghatchian J. An overview of current position on cell therapy in transfusion science and medicine: from fictional promises to factual and perspectives from red cell substitution to stem cell therapy. Transfus Apher Sci 2020;59;102940.

[36] Katz LM. (A little) clarity on convalescent plasma for Covid-19. N Engl J Med 2021;384;666-8.

[37] Joyner MJ, Scott Wright R, Fairweather D, Senefeld JW, Bruno KA, Klassen SA, et al. Early safety indicators of COVID-19 convalescent plasma in 5000 patients. J Clin Invest 2020;130; 4791-7.

[38] Tobian AAR, Shaz BH. Earlier the better: convalescent plasma. Blood 2020;136;652-4.

[39] Cantini F, Goletti D, Petrone L, Fard SN, Niccoli L, Foti R. Immune therapy, or antiviral therapy, or both for COVID-19: a systematic review. Drugs 2020;80;1929-46.

[40] Mirtaleb MS, Mirtaleb AH, Nosrati H, Heshmatnia J, Falak R, Emameh RZ. Potential therapeutic agents to COVID-19: an update review on antiviral therapy, immunotherapy, and cell therapy. Biomed Pharmacother 2021;138;111518.

[41] Izda V, Jeffries MA, Sawalha AH. COVID-19: a review of therapeutic strategies and vaccine candidates. Clin Immunol 2021;222;108634.

[42] Liu STH, Lin HM, Baine I, Wajnberg A, Gumprecht JP, Rahman F, et al. Convalescent plasma treatment of severe COVID-19: a propensity score-matched control study. Nat Med 2020;26;1708-13.

[43] Leng Z, Zhu R, Hou W, Feng Y, Yang Y, Han Q, et al. Transplantation of ACE2- mesenchymal stem cells improves the outcome of patients with COVID-19 pneumonia. Aging Dis $2020 ; 11 ; 216-28$. 